\title{
Espacios de Formación Integral: transformaciones y desafíos para la enseñanza y la docencia de Ciencias Económicas y Administración
}

\author{
Jimena Castillo \\ Unidad de Extensión y \\ Relacionamiento con el Medio. \\ uerm@ccee.edu.uy
}

Natalia H. Correa

Unidad de Apoyo a la Enseñanza.

ncorrea@ccee.edu.uy

Varenka Parentelli

Unidad de Apoyo a la Enseñanza.

vparentelli@ccee.edu.uy

\author{
Melina Romero \\ Departamento de Ciencias de \\ la Administración. \\ melina.romero@ccee.edu.uy \\ Facultad de Ciencias Económicas y \\ de Administración de la Universidad \\ de la República, Uruguay.
}

A 100 años de la Reforma Universitaria de 1918

Desafíos de gestión

RECEPCIÓN: 24/06/17

ACEPTACIÓN FINAL: 23/10/17

\section{Resumen}

Con la "Segunda Reforma Universitaria" se impulsa en la Universidad de la República (Uruguay) la curricularización de la extensión y su articulación con las otras funciones universitarias. Esto impulsó las propuestas educativas en torno a los Espacios de Formación Integral (EFI) que promueven la autonomía del estudiante, el pensamiento crítico y la interdisciplina, entre otros aspectos. En la Facultad de Ciencias Económicas y de Administración (FCEA) los EFI integran la oferta de unidades curriculares opcionales del Plan de Estudios 2012. En este contexto se visualiza la necesidad de tener docentes formados en esta nueva modalidad de enseñanza, lo que a su vez supone un reto para el docente y para la institución. Este trabajo tuvo como objetivos conocer si los docentes experimentan algún tipo de cambio al transitar por los EFI e identificar si reconocen modalidades pedagógicas alternativas, así como realizar una aproximación sobre sus concepciones y valoraciones al respecto.

\section{Palabras clave}

- Espacios de Formación Integral

- Modalidades pedagógicas alternativas

- Concepciones docentes

- Desafíos en la docencia universitaria

\section{Resumo}

Com a "Segunda Reforma Universitária" a inclusão da extensão no curriculo e suaarticulaçãocomoutrasfunçõesuniversitáriassão promovidas na Universidad de la República (Uruguay). Issoimpulsionou as propostas educativas em torno dos espaços de formação integral (EFI) que promovemautonomia do aluno, o pensamento crítico e interdisciplinar, entre outros. Na Facultad de Ciencias Económicas y de Administración (FCEA) os EFI passam a integrar a oferta de unidades curriculares opcionais do curriculum 2012. Neste contexto, a necessidade de professorestreinadosnesta nova modalidade de ensino é observada, o que por sua vez é umdesafio para os professores e para a instituição. Este trabalhofoi criado com os objetivos de conhecer se os professoresexperimentamalgum tipo de mudança no trânsitoatravés dos EFI e identificar se eles reconhecem métodos de ensino alternativos. Por último, se fezumaabordagem sobre suasconcepções e avaliações sobre o assunto.

Palavras-chave

- Espaços de Formação Integral

- Métodos de ensino alternativos

- Concepções docentes

- Desafios no ensino universitário
Para citación de este artículo

Castillo, J., Correa, N. H., Parentelli, V. y Romero, M. (2017). Espacios de Formación Integral: transformaciones y desafíos para la enseñanza y la docencia de Ciencias Económicas y Administración. . Revista $+E$ versión en línea, 7(7), 156-163. Santa Fe, Argentina: Ediciones UNL. 


\section{Introducción}

Los docentes universitarios configuran su rol e identidad como tales a partir de tres grandes dimensiones: la laboral, la personal y la profesional (Zabalza, 2007). Las condiciones de trabajo, las funciones que se deben desarrollar, la formación necesaria disciplinar, didáctica, metodológica - para cumplir adecuadamente con las tareas, entre muchos otros aspectos, poseen una influencia sustantiva en la construcción de sus concepciones respecto de su papel en la institución. De acuerdo con los propósitos de esta investigación, y dada la complejidad de actividades implicadas y el conjunto de saberes necesarios para su cumplimiento, se entiende a la docencia de nivel universitario como una profesión polivalente y peculiar (Perera, 2006; Collazo, 2008) y al docente universitario como aquel académico que desarrolla funciones de enseñanza, investigación, extensión y gestión (Errandonea, 1998). Claramente, y dependiendo de las condiciones de trabajo -niveles de responsabilidad, asignaciones horarias-, un docente universitario podrá asumir y cumplir dichas funciones con mayor o menor grado de desarrollo.

En cuanto a la función formativa del profesorado universitario, García-Valcárcel (2001) plantea que los docentes poseen dos roles en tensión. En primer lugar, un rol asignado y asumido por tradición: el docente se encuentra en posesión de un saber que debe transmitir pues domina la disciplina. En segundo lugar, un rol nuevo y que la sociedad demanda: el docente ya no es la única fuente de información.

En la Universidad de la República (UdelaR), la Segunda Reforma Universitaria ${ }^{1}$ (Arocena, 2011) desarrolló políticas de promoción de la extensión y la integralidad que incluyeron la curricularización de la extensión reconociéndola como una instancia más de formación. En dicho marco surgen los Espacios de Formación Integral (EFI), que tienen como propósitos articular las funciones universitarias, promover la autonomía del estudiante, el pensamiento crítico y propositivo, así como fomentar el trabajo en grupo desde una perspectiva interdisciplinaria.

Los EFI fomentan el vínculo entre el aprendizaje y la interacción con la sociedad. Se llevan adelante mediante cursos teóricoprácticos dirigidos a estudiantes de grado. Funcionan generalmente bajo modalidad de taller y tienen como particularidad el aprendizaje fuera del salón de clases. Los estudiantes cuentan con una formación teórica tanto en lo que refiere al trabajo con sujetos no universitarios, como en el área específica en la que desarrollarán la práctica, previa y durante el trabajo de campo. Todo el trabajo práctico es acompañado y supervisado por el docente, que oficia de guía y tutor, pero donde el protagonismo lo tienen los estudiantes. En estos espacios se favorece el intercambio de conocimientos entre universitarios y no universitarios en tanto se reconoce la pluralidad de saberes, la integración de equipos interdisciplinarios como forma de aproximarse de manera más acertada a los problemas en el entendido de que la realidad es compleja y la combinación de enseñanza, investigación y extensión en la metodología de trabajo propuesta.

En la Facultad de Ciencias Económicas y de Administración (FCEA), los EFI integran la oferta de unidades curriculares (UC) opcionales del Plan de Estudios (PE) 2012, asignando créditos como actividades integradoras de dicho Plan. En líneas generales, los EFI se encuentran vinculados al trabajo con el sector productivo, cooperativismo, micro empresas, organizaciones sociales sin fines de lucro, creación y gestión colectiva de centros culturales y comunitarios, fortalecimiento con el sector público, redes y desarrollo local.

Si bien los EFI no son actividades obligatorias, los estudiantes deben contar con un mínimo de créditos en el área integradora y en algunas carreras de la FCEA la única oferta para esta área son estos espacios formativos. Por la modalidad de trabajo se debe limitar la cantidad de estudiantes que participan, por lo que no todos pueden acceder ni cumplir con los créditos requeridos por el PE para el área integradora. Esto tiene como consecuencia el rezago de algunos estudiantes que redunda en una tensión entre lo que el PE requiere y la oferta que la facultad realiza. A tales efectos la institución debió gestionar algunos dispositivos en torno a dar prioridad, en el sorteo para cubrir los cupos, de aquellos estudiantes avanzados en la carrera.

Por otra parte el financiamiento de estas UC proviene del Servicio Central de Extensión y Actividades en el Medio (SCEAM), por lo que en caso de limitarse o finalizarse ese financiamiento, la FCEA no estaría en condiciones de asumirlo y peligraría la continuidad de los mismos. Esto genera otra tensión con los requisitos del PE. A lo anterior se suma el hecho de que en la discusión de las asignaciones de los recursos económicos con respecto a las horas docentes, no están contempladas las particularidades de los EFI en cuanto a la responsabilidad y tiempo de dedicación que implican para la docencia. Esto hace que se distribuyan los recursos sobre el criterio de los cursos "tradicionales", que toma como referencia principal para la asignación de horas docentes a la cantidad de estudiantes y no tienen en cuenta aspectos relacionados al trabajo con actores no universitarios y el seguimiento continuo de las tareas de campo de los estudiantes, entre otros aspectos. Asimismo, existe otra tensión con respecto a la posibilidad de 
generar oferta y la formación docente, ya que no todo el profesorado está capacitado ni dispuesto para incluir modalidades alternativas de enseñanza en sus propuestas pedagógicas. Si bien lo anterior es una responsabilidad de los docentes también lo es de la institución, ya que debe motivarlos y establecer políticas institucionales acordes a las demandas formativas.

Conocer los referentes que utilizan los docentes en su actividad, y en particular sus representaciones en cuanto al trabajo en los $\mathrm{EFI}$, resulta de interés debido a que — de acuerdo con la abundante literatura disponible - hay una relación entre los significados que los profesores otorgan a su actividad y las acciones que emprenden (Feixas, 2010).

En este marco, el presente artículo presenta algunos resultados del proyecto de investigación "Transformaciones en la docencia: el caso de los Espacios de Formación Integral en la Facultad de Ciencias Económicas y de Administración", realizado entre la Unidad de Extensión y Relacionamiento con el Medio (UERM) y la Unidad de Apoyo a la Enseñanza (UAE) de la FCEA, el cual tuvo como principales objetivos:

- Conocer si los docentes experimentan algún tipo de cambio al transitar por los EFI, identificarlos y describirlos.

- Identificar si los docentes reconocen, en su experiencia en los EFI, modalidades pedagógicas alternativas y realizar una aproximación sobre la valoración que hacen al respecto.

- Generar insumos para la toma de decisiones institucionales, administrativas y de gestión educativa.

Dicho proyecto da continuidad a un trabajo realizado en 2014 por la (UERM) sobre las percepciones de los estudiantes acerca de los EFI (Castillo, Rivero y Romero, 2014). El mismo plantea la necesidad de profundizar en la indagación mediante consultas a los docentes sobre su experiencia a partir de la tarea en los $\mathrm{EFI}$, así como avanzar luego en un relevamiento a los actores no universitarios que participan de dichas experiencias, abarcando de esta manera la mirada de todas las partes que conforman estas prácticas formativas.

\section{Abordaje metodológico}

Se optó por un abordaje metodológico cualitativo y exploratorio, sobre la base de entrevistas individuales semiestructuradas. De los 12 docentes de la FCEA que fueron referentes de un EFI en más de una ocasión entre 2010 y 2015 inclusive, fueron entrevistados 10. La pauta de entrevista consistió en un guion orientador en el que se buscó indagar las concepciones de los entrevistados sobre los aspectos centrales de los EFI como propuestas formativas, los modos de abordar los contenidos, el relacionamiento docentesestudiantes-comunidad y los posibles cambios en la enseñanza. El análisis se desarrolló en diversas etapas: 1) definición del segmento con significado como unidad de análisis; 2) reconocimiento de dichas unidades en las entrevistas y su codificación; 3) recuperación de los segmentos codificados con las categorías surgidas y, utilizando el método comparativo constante, contrastación y precisión de las mismas; 4) organización de las categorías en grandes temas.

Luego de dicho proceso, se organizó la información en tres grandes categorías: por un lado, los aspectos vinculados a la enseñanza en tanto actividad; por otro, los vinculados a la docencia en tanto profesión y, por último, los aspectos atinentes a vivencias personales:

1. "Enseñanza". Se identificaron dos propiedades con sus respectivas dimensiones. La propiedad "relación pedagógica" alude a la "tríada didáctica ampliada", donde aparece el actor no universitario como cuarto componente. Dimensiones: el lugar del saber (saberes populares, disciplinares e interdisciplina), el vínculo con los estudiantes (en lo afectivo, en lo didáctico y lo comunicacional), los aprendizajes (de estudiantes y docentes en el trabajo con actores no universitarios) y el rol docente. En cuanto a la propiedad "actividades didácticas", se incluyen dimensiones respecto de la tarea de enseñar, tales como la planificación, las metodologías o estrategias de enseñanza, los recursos didácticos, la evaluación y la relación teoría-práctica.

2. "Docencia" (como profesión). Incorpora las comparaciones de los entrevistados sobre su experiencia en otras formas de organización del trabajo en los equipos docentes que integran fuera de los EFI, de tipo más "tradicional", las posibilidades de aportar al diseño curricular, la autonomía en la actuación, entre otros.

3. "Vivencias personales". Da cuenta de las reflexiones docentes sobre los cambios por los cuales transitaron a nivel personal a partir de la experiencia en el EFI. Se consideraron expresiones de sentimientos explicitados con relación al ejercicio de la enseñanza en torno a factores motivacionales, evolución, entre otros.

En cuanto a los aspectos emergentes, considerados como transversales a las categorías anteriores, se recuperó lo referente a los cambios que surgen alrededor de la participación en el EFI que afecta el ejercicio de la docencia. Algunos de ellos son: cuestionamientos sobre las condiciones laborales, integración a equipos docentes, lugar de desarrollo de la práctica de enseñanza. Si bien esta investigación no tuvo como propósito realizar un análisis comparativo entre los EFI y la forma "tradicional" de enseñanza, en el correr de las entrevistas los docentes hacen mención a ambas modalidades, las comparan y hacen comentarios valorativos. Este emergente es tomado por el equipo investigador e incluido en el análisis y presentación de resultados por considerarlo de importancia debido al énfasis que los mismos docentes pusieron en eso.

\section{Análisis de los resultados}

Para este artículo se presentarán los resultados haciendo foco en las categorías referidas a los desafíos de la docencia en los EFI. 
En los EFI, el lugar del docente en la planificación del curso resulta un desafío. Se destaca la flexibilidad de estos espacios para amoldarse a las situaciones que surgen a lo largo de su implementación

\section{La tríada didáctica ampliada: cambios en la relación pedagógica}

Por "tríada didáctica ampliada" entendemos aquellas configuraciones de aula en las que aparece el actor no universitario como cuarto componente. En este sentido, la tríada didáctica como configuración "tradicional" de aula —relación docenteestudiante-conocimiento- no permite analizar los fenómenos educativos que se producen en los EFI. La conceptualización surge con las reflexiones de los docentes entrevistados acerca de las relaciones entre los actores y los saberes involucrados en las situaciones de enseñanza propias de estos espacios.

Respecto del lugar del conocimiento, según los entrevistados se produce un descentramiento de la figura del docente como única fuente del saber, quien igualmente aprende a partir del saber popular que comparten los actores no universitarios.

El vínculo con los estudiantes también se ve modificado. En este sentido, mediante estas prácticas se producen cambios en la forma de enseñar y se reafirma una concepción cercana a un proceso participativo del estudiante y de aprendizaje desde el contacto con la realidad. Los entrevistados señalan un estudiante activo, más involucrado con el curso y con mayor grado de autonomía, manifestándose vínculos más horizontales y una relación de confianza, en contraposición con las formas más "tradicionales" de enseñanza en las que se observa una relación más vertical. El descentramiento de la figura del docente como única fuente de información y las modificaciones en el vínculo con los estudiantes interpelan el papel del profesorado. El docente, entonces, adquiere un rol de liderazgo y acompañamiento, distinto del rol de transmisor de conocimientos en un formato más "tradicional" de enseñanza. Según los entrevistados, es parte de su rol la construcción de vínculos y de confianza con los estudiantes a partir de la escucha y de un trato horizontal. Así, se identifica a un docente flexible y articulador, que enriquece en muchos casos la concepción sobre la docencia:

"La interdisciplina requiere de una apertura y de interés en ver cómo articular saberes, eso se tiene, podés tenerlo o no tenerlo y en el caso de los EFI creo que aporta bastante". (Entrevistado 1)

"Cambió mi rol y mi forma de relacionarme con la enseñanza, como me represento como docente". (Entrevistado 2)

\section{La enseñanza en los EFI: transformaciones en las actividades didácticas}

Si bien la planificación, la evaluación, la definición de estrategias de enseñanza y la selección de recursos didácticos son actividades típicas de la docencia, los entrevistados manifiestan que su trabajo en los EFI es diferente del que realizan en cursos "tradicionales". En los EFI, el lugar del docente en la planificación del curso resulta un desafío. Se identifica una participación activa de todos los docentes del EFI en la definición del curso y su desarrollo. Se destaca la flexibilidad de estos espacios para amoldarse a las situaciones que surgen a lo largo de su implementación. Los EFI exigen una revisión y actualización permanente. Al respecto, uno de los entrevistados manifiesta:

"Acá vas a ver con qué te encontrás, qué problema tuvieron los estudiantes, qué dificultad te plantean, entonces no es que vayas con un plancito clase a clase". (Entrevistado 3)

Los entrevistados plantean que los cursos "tradicionales" se han estandarizado y estructurado. Además, los docentes que se 
encuentran en la base de la pirámide académica tienen poca o nula incidencia en su diseño e implementación. Así, la posibilidad que tienen los docentes en los EFI de aportar a la planificación se traduce en lo que definen como "libertad" y fomenta una actitud receptiva al cambio y proactiva, que habilita la toma de decisiones:

"Pero después de estar muchos años con una metodología de trabajo igual y sin poder tener mucha incidencia te genera un proceso de una palabra que es achanchamiento". ${ }^{2}$

(Entrevistado 4)

Si bien los docentes cuentan con un contrato didáctico institucional, que establece pautas y ponderadores en función de los objetivos de enseñanza, la evaluación es considerada como una de las etapas más complejas en este tipo de espacios formativos. Es comprendida como un desafío ya que debe definir una evaluación continua y formativa, además de considerar tanto el trabajo en equipo como el proceso individual. Para ilustrar lo anterior destacamos las siguientes reflexiones realizadas por dos docentes:

"Más complejo que evaluar una materia común, que tienen dos parciales, un control de lectura y no mucho más (...) por ejemplo, ahora estoy corrigiendo parciales en una materia y solo son dos parciales, lo único que se evalúa son los parciales y, por ejemplo, no es lo mismo una persona que nunca vino y solo vino a los parciales con uno que vino a todas las clases, que participó de todos los prácticos". (Entrevistado 5)

"La calificación no es un premio a la buena voluntad, aunque el compromiso es relevante, por ahí son un montón de elementos, que no sería demasiado justo que quedaran exclusivamente en la percepción personal, que puede estar muy impregnada de simpatías". (Entrevistado 1)

En cuanto a lo metodológico, los docentes incluyen en los EFI estrategias de enseñanza diferenciadas con respecto a lo "tradicional". Estos espacios formativos se caracterizan por incluir dinámicas de talleres y trabajos dentro y fuera del salón de clase. Cabe hacer notar que de las entrevistas emerge que, como consecuencia de la experiencia en los EFI, el uso de estas metodologías por parte de los docentes trasciende estos espacios puesto que en sus clases "tradicionales" incorporan este tipo de estrategias. Debe tenerse en cuenta que muchas de las unidades curriculares de la FCEA no son numerosas y sin embargo los docentes adoptan una modalidad expositiva "tradicional", y así lo expresan:
"A partir de que trabajo en los EFI trato muchísimo más de hacer trabajar al estudiante en las clases. Antes era más de dar clases magistrales, y después de los EFI empecé a acentuar más el trabajo de ida y vuelta y de hacerlos trabajar a ellos". (Entrevistado 6)

"Tratar de aplicar un poco más la mecánica de taller digamos en las clases. Más que nada en algunas clases que son unos 30 y pocos y utilizar dinámicas que antes no utilizaba tanto hasta que me empecé a informar, empecé a hacer (el EFI) como la parte que no estaba formado en eso". (Entrevistado 5)

Este tipo de actividades formativas, por sus características constitutivas, requiere una actualización permanente de los recursos didácticos. Y los docentes ponen énfasis en este aspecto, ya que lo identifican como fundamental. Asimismo, surge de las entrevistas la importancia del uso del Entorno Virtual de Aprendizaje (EVA) como recurso didáctico a fin de fortalecer el vínculo con el estudiante y la conexión entre encuentros. Es ilustrativa la definición que un docente hace sobre lo "tradicional" como "aula, pizarrón y teoría" (Entrevistado 8) en contraposición a los recursos didácticos utilizados en los EFI.

Sobre la relación teoría-práctica en la enseñanza, en los EFI el componente práctico es considerado fundamental debido a que la actividad formativa se encuentra en un contexto real, orientada y supervisada por un docente referente. En este marco, se hace mención a la aplicación de conceptos teóricos previamente abordados y al trabajo con nuevos conceptos durante las distintas actividades en campo. Esto permite cotejar la teoría con la realidad: "Lo otro que me parece central son las prácticas en el campo. Eso me parece que está muy bueno y le da un toque muy distinto a lo que en realidad son el resto de las materias, porque que yo conozca no hay tantas materias en facultad que los estudiantes estudien y apliquen. Lo aplican en la vida, en ellos profesionalmente cuando empiezan a trabajar... pero que la facultad luego lo evalúe aplicándolo en la sociedad, me parece central”. (Entrevistado 3)

\section{La docencia como profesión: cambios en las formas de trabajo} En contraposición a algunas formas de trabajo arraigadas en la institución, como ya se mencionó, los entrevistados plantean una mayor autonomía en su actividad en los EFI. Así, estos espacios son visualizados como flexibles, donde el docente tiene libertad de acción y, por tanto, son vistos como espacios democratizadores de la enseñanza. El trabajo en los EFI es identificado como desafiante, 


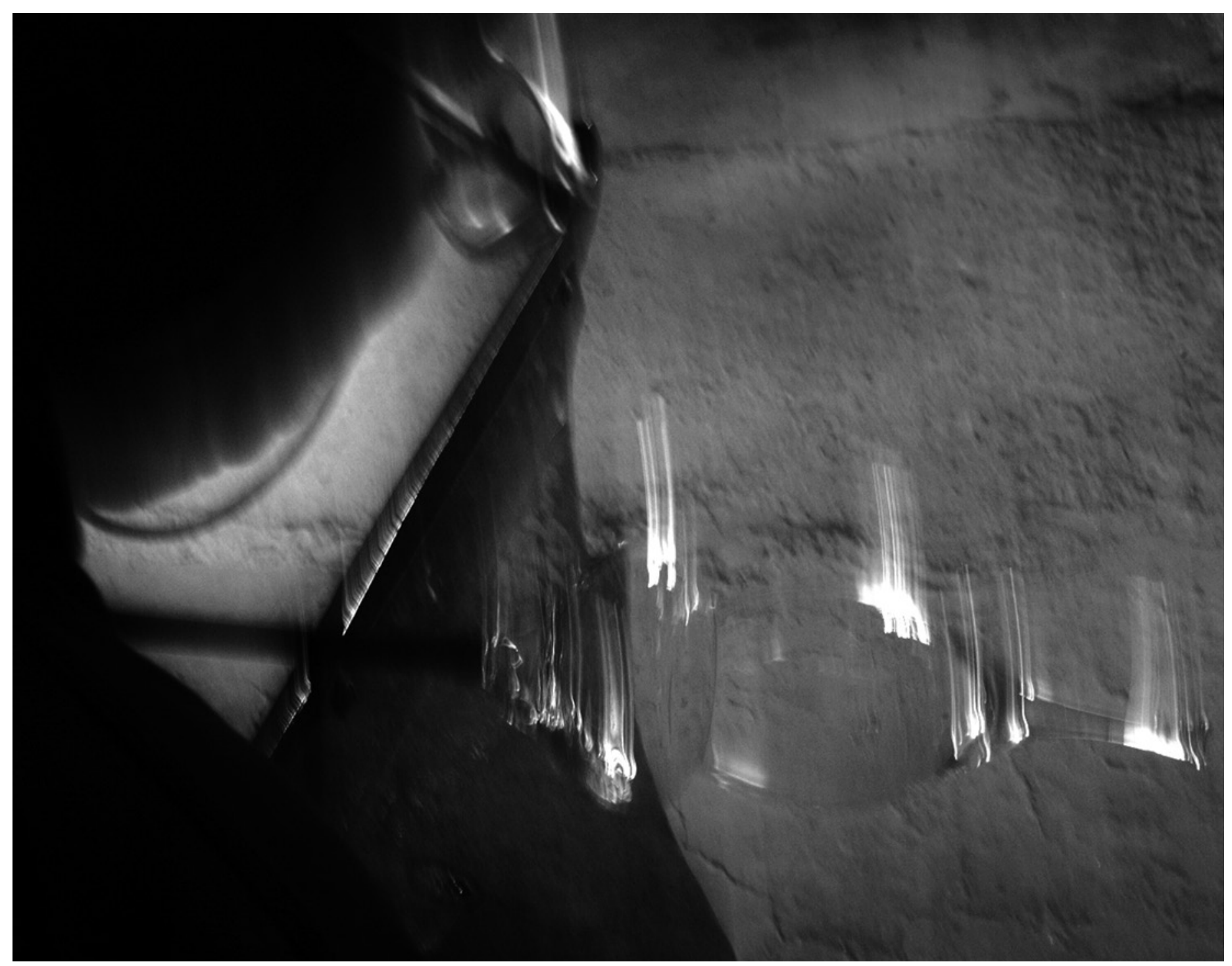

(c) Oscar Dechiara

\section{G6}

El trabajo en los EFI es identificado como desafiante, interdisciplinario, con relaciones horizontales entre los actores involucrados y donde se valora particularmente el trabajo en campo 
interdisciplinario, con relaciones horizontales entre los actores involucrados y donde se valora particularmente el trabajo en campo:

"democratiza la enseñanza, conlleva riesgos de equivocarse y desalinearse de las pautas, es un tema inherente a ser más partícipe del diseño del proyecto de enseñanza. Es un riesgo que vale la pena pagar". (Entrevistado 8)

"Existen múltiples actores, están las contrapartes, están los beneficiarios, en algún caso, es muy flexible, entonces los docentes tienen que tener apertura a eso, y me parece que no cualquiera, no porque se necesite nada especial, sino por la apertura misma". (Entrevistado 2)

La poca posibilidad de incidir en el proyecto de enseñanza de su unidad curricular de base y las diferencias de poder que genera la estructura vertical a la que anteriormente se ha referido traen aparejada una crisis con la metodología "tradicional" de enseñanza. Esto interpela a los profesores, quienes se cuestionan la forma de trabajo y expresan descontento con su profesión docente tal como venía funcionando hasta ahora. En algunos casos, dicha crisis es tal que algunos entrevistados han decidido no continuar trabajando en cursos "tradicionales":

"La sensación de eslabón, la cadena, y con muy poca chance de incidir, siendo hasta grado dos no sentía que mi participación fuera muy activa. Después de la experiencia me resultó muy difícil retomar la enseñanza de ese punto de vista. Ahora directamente no podría". (Entrevistado 4)

"Por suerte ya no doy más cursos tan estandarizados y bueno, yo me acomodo a un contenido específico y me gusta más ese tipo de cursos, donde yo soy quien propone activamente". (Entrevistado 2)

Sumado a lo anterior, algunos docentes manifiestan la necesidad de contar con mayor interacción en sus otras unidades curriculares y valoran el vínculo y la integración con la Unidad de Extensión y Relacionamiento con el Medio (UERM):

"Me gustaría sí que tuviéramos una devolución más abierta (...) el feedback nunca me llega, nunca me entero (...) que se reúna contigo o quien sea; 'tenés esto, esto es tu resultado, acá estás mal', no sé, un tirón de orejas, una felicitación, lo que venga, pero nada, no te enterás de nada. No sé si es nuestro caso o es en general (...) Acá la verdad que tenemos más seguimiento, con las chicas de Extensión, la verdad que hay mucho más contacto, pero en las otras cátedras". (Entrevistado 3)

\section{La dimensión personal: desafíos, motivación y enriquecimiento}

En esta dimensión se habla de una evolución personal a partir del pasaje por el EFI, de un antes y un después. Es de destacar que en los discursos de los entrevistados aparecen explícitos sentimientos y emociones asociados a la enseñanza y al aprendizaje de los estudiantes, como puede visualizarse en los siguientes comentarios: "Porque es un placer ver cómo los estudiantes se desempeñan con uno, hablando con la contraparte, es un placer ver como ellos tienen la capacidad para aprender, uno lo que tiene que hacer es guiarlos un poco. Descubrir cosas sobre los estudiantes que no tenías idea". (Entrevistado 6)

"Esto me muestra que se puede trabajar diferente, que tengo mucho para dar, que del otro lado hay una buena valoración, entonces uno aporta, es lo que me mantiene en armonía". (Entrevistado 7)

Del mismo modo, se plantean cuestiones tales como la plenitud en la tarea, el placer en el desarrollo de la profesión docente, el desafío constante, la motivación y el enriquecimiento personal:

"Como docente no sé especificar claramente qué le debo a los EFI pero hay una cuota, parte importante que tiene que ver con cómo me representó como docente, qué cambió en mi historia, y que eso coevolucionó con los EFI”. (Entrevistado 2)

"para mí fue una experiencia riquísima porque te da para repensar todo lo que venía siendo mi rol como docente en la facultad". (Entrevistado 4)

"yo me siento cada vez más plena en la tarea, siento que voy evolucionando, que es muy desafiante (...) estoy sumamente motivada y agradecida por la experiencia". (Entrevistado 7)

\section{Conclusiones}

"Pero sí hubo un antes y un después de haber tenido la oportunidad de pasar por el EFI". (Entrevistado 4)

Luego de analizadas las entrevistas, se puede afirmar que los docentes manifiestan que se produce un cambio en sus concepciones a partir de la participación en los EFI. Cabe mencionar además que, a partir de la experiencia por la cual transitaron en el marco de estos espacios formativos, los docentes son capaces de realizar estas reflexiones e incluso contar con un 
marco comparativo. A este respecto, se reconocen modalidades pedagógicas alternativas, sobre todo cuando la comparan con la modalidad "tradicional"; plantean que el modo de relacionarse con el estudiante es otro y destacan la importancia de la interacción con el actor comunitario y su saber no académico. Para ellos existe una reconfiguración sobre la forma de entender la enseñanza y el aprendizaje en cuanto a que el aula se magnifica por la participación de distintos actores y la articulación de saberes académicos y populares. A su vez, el docente de un EFI se identifica como protagonista de la estrategia pedagógica, lo que valora positivamente. Sin embargo, y aunque todos los entrevistados manifiestan que recomendarían el trabajo en un EFI a otros colegas, enfatizan sobre la necesidad de un perfil docente que cumpla con ciertas características que posibiliten transitar efectivamente por la experiencia, teniendo en cuenta que se trata de una modalidad de enseñanza y de aprendizaje diferente. Como se mencionó al inicio, los EFI son actividades formativas curricularizadas y, si bien responden a características disímiles de lo que se conoce como "tradicional", acreditan como cualquier otra propuesta enmarcada en el currículo. No obstante, Ilama la atención que algunos docentes no identifican a los EFI como unidades curriculares, a pesar de saber que los mismos son UC opcionales de las carreras que brinda la FCEA. Es oportuno hacer notar que los docentes que participan en los EFI lo hacen porque los han invitado o se han acercado voluntariamente en búsqueda de modalidades alternativas y con características formativas diferenciadas de lo que ellos Ilaman "tradicional". Es tal el grado de diferenciación que hacen entre "lo alternativo" y lo "tradicional" que no logran ubicar estas modalidades como espacios formativos curriculares. En definitiva, esto permite hacer visible una realidad institucional implícita dada por una cultura académica dominante en torno a lo "tradicional" que refiere a metodologías exclusivamente expositivas en las clases y, por ejemplo, la verticalidad de la estructura y la dinámica de trabajo de los docentes. En contraposición, los EFI emergen como espacios "alternativos" donde el conocimiento se construye en interacción entre distintos actores y los docentes asumen roles más protagónicos en la toma de decisiones.

Otro aspecto a destacar refiere a la identificación que los docentes realizan acerca de la necesidad de una actualización permanente de los recursos y estrategias didácticas incluidas en los EFI. Respecto de este tema, por el énfasis que hacen en estos asuntos como característicos de los EFI, podría inferirse que esta necesidad no es identificada como tal para las unidades curriculares "tradicionales".
Todo lo anterior, con relación a las tensiones, la no identificación de estos espacios como curriculares y la necesidad de que el docente cumpla con características específicas para llevar adelante estos espacios, indica que se requiere que la institución apoye a los docentes y brinde espacios de formación pedagógica que permitan reflexionar sobre la enseñanza y el aprendizaje en los EFI.

\section{Referencias bibliográficas}

Arocena, R. (2011). Curricularización de la extensión: ¿por qué, cuál, cómo? En Cuadernos de Extensión. Integralidad. Tensiones y Perspectivas, 1, 9-19. Montevideo. Castillo, J.; Rivero, A.; Romero, M. (2014). Avances en la institucionalización de la extensión y la integralidad en la FCEA: 2009-2014. Recuperado de: http://eva.udelar.edu.uy/pluginfile.php/507878/mod_resource/content/1/Extracto\%20UERM.pdf. Collazo, M. (2008). El sentido de la Didáctica en la formación docente universitaria. En Universidad de la República, Comisión Sectorial de Enseñanza, Debates teóricos, metodológicos y políticos sobre la formación docente universitaria. Montevideo: Universidad de la República, Comisión Sectorial de Enseñanza.

Errandonea, A. (1998). La Universidad en la encrucijada. Hacia otro modelo de Universidad. Montevideo: Nordan Comunidad.

Feixas, M. (2010). Enfoques y concepciones docentes en la universidad. RELIEVE. Revista Electrónica de Investigación y Evaluación Educativa, 16(2), 1-27. Recuperado de: http://www.uv.es/relieve/v16n2/RELIEVEv16n2_2.htm

García-Valcárcel, A. (2001). La función docente del profesor universitario, su formación y desarrollo profesional. En A. García-Valcárcel (Coord.), Didáctica universitaria (pp. 9-43). Madrid: La Muralla.

Perera, H. (2006). Formación permanente del docente universitario: una experiencia en el Área Social de la UdelaR. Conversación, 34-43.

Zabalza, M. A. (2007). La enseñanza universitaria: El escenario y sus protagonistas. Madrid: Narcea. 